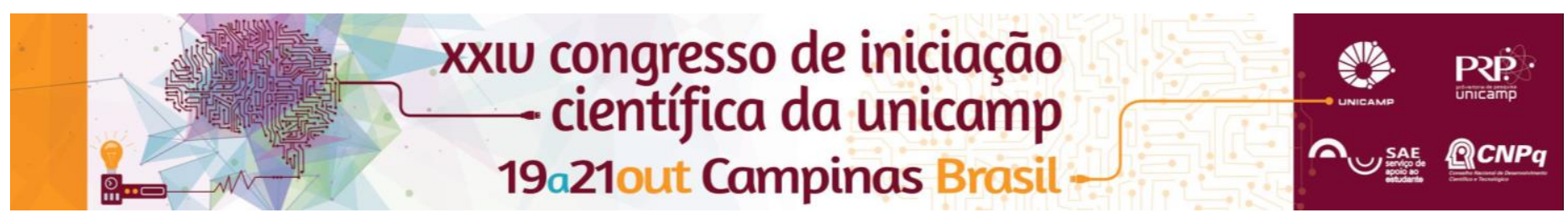

\title{
Solution-processable Blue-Greenish Hybrid Polymeric/Perovskites Quantum Dots Light Emitting Diodes
}

\author{
Glauco M. Santiago, José Carlos Germino, Jilian Nei de Freitas, Emre Yassitepe, Teresa D. Z. Atvars and Ana \\ Flávia Nogueira
}

\section{Abstract}

In this present research the applicability of quantum dot (CsPbBr3 perovskite) and polymer nanocomposites in LED were studied. Photophysical properties of the materials and the final hybrid LED were reached. The purpose of this material combination is the desirable properties mix of both.

\section{Key words:}

Perovskites, polymers, HLEDs

\section{Introduction}

Currently the organic electronic is growing, especially in light emitting diodes. This kind of material can be casted in flexible substrates. ${ }^{1}$ The main problems in organic LEDs are low efficiency compared with inorganic ones.

This failure can be avoided by adding inorganic materials with good luminescence, and preserving the processability in flexible substrates.

In this scenario a new material aroused with promising properties. $^{2}$ The Cesium Lead Halide perovskites $\left(\mathrm{CsPbX}_{3}\right)$ have emission that depend on the halide $(\mathrm{X})$, varying from the blue (chlorine) to red (lode). This material can be processed in solution with a polymer to be casted in a substrate. In this current research was combined a polymer with $\mathrm{CsPbBr}_{3}$ (green perovskite) in solution casted in films on a substrate to manufacture the devices.

\section{Results and Discussion}

It was fulfilled the photophysical measurements of different film conformation, all including a nanocomposite of quantum dots and polymer. At first the films were casted with quantum dots and Poly(9-vinylcarbazole) (PVK).

The absorbance in both $\mathrm{CsPbBr}_{3}$ and $\mathrm{PVK}$ films are showed as peaks near of $290 \mathrm{~nm}$, more intensely in the perovskite one. Films with the nanocomposite have intermediate absorbance between QD and polymer. Making use of this data, the films were excited at $290 \mathrm{~nm}$ and the fluorescence spectra were obtained.

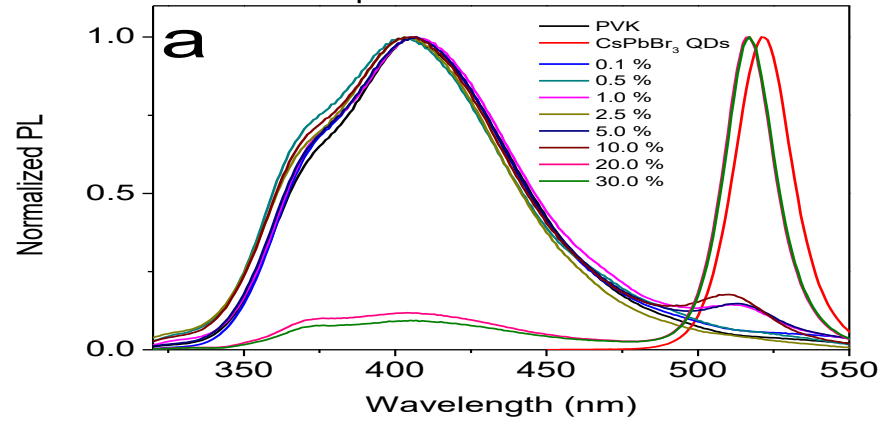

Image 1. Photoluminescence of PVK, QD and nanocomposites at 290nm excitation.

It is seen that PVK presents emission in $407 \mathrm{~nm}$, while the typical perovskite emission is $521 \mathrm{~nm}$. In the composite films as great as the proportion of perovskite was, the peak in $407 \mathrm{~nm}$ had its intensity reduced and the quantum dot typical emission increased the intensity. Starting from $20 \%$ $(w / w)$ of quantum dot in the film, the emission of PVK can be considered negligible and perovskite appears strongly. The composite solutions were applied to fabricate the HLEDs, whose the composition is Glass|ITO |PEDOT:PSS|PVK:PQDs (\% w/w)|Ca|Al



Image 2. Electroluminescence of HLEDs.

The electroluminescence spectra show about the same behavior of the photoluminescense. However, it seems that the PVK and $\mathrm{CsPbBr}_{3}$ compete for charges, because PVK does not "disappear" as in photoluminescence. The results are not so pure spectra, nor blue neither green, which is expected for a only PVK or Quantum Dot LED, or with a complete energy transfer between the materials. The resume of optical-electronic properties of the PVK:PQDs composites HLEDs is shown below.

Chart 1. Optical-electronic properties of the PVK:PQDs composites HLEDs.

\begin{tabular}{cccc}
\hline & $L_{\max } / \mathbf{c d ~ m}^{-2}$ & $\mathrm{~J} \mathbf{A ~ c m}^{-2}$ & $\eta_{\max } / \mathbf{m c d ~ A}^{-\mathbf{1}}$ \\
\hline $\mathbf{5 . 0} \%$ & 31.0 & 0.49 & 8.6 \\
$\mathbf{1 0 . 0} \%$ & 10.0 & 0.84 & 1.6 \\
$\mathbf{2 0 . 0} \%$ & 9.0 & 0.83 & 0.5 \\
$\mathbf{3 0 . 0} \%$ & 9.5 & 0.46 & 2.2 \\
\hline
\end{tabular}

Conclusions

The HLEDs achieved in this research cannot be used in a emission of monochromatic light. The present HLEDs show a combination of blue and green emission that combined with a red emitting material can show a white emission.

\section{Acknowledgement}

The authors thank SAE, Fapesp, CNPq and Capes for financial support and fellowships.

\footnotetext{
${ }_{1}$ Akcelrud, L. Prog. Polym. Sci. 2003, 28 (6), 875-962.

2 Protesescu, L.; Yakunin, S.; Bodnarchuk, M. I.; Krieg, F.; Caputo, R.; Hendon, C. H.; Yang, R. X.; Walsh, A.; Kovalenko, M. V. Nano Lett. 2015, 15 (6), pp 3692-3696.
} 